\title{
CJEM Debate Series: \#DomesticViolence - We should routinely screen for domestic violence (intimate partner violence) in the emergency department
}

\author{
Nikita Arora, BHSc, MD*; Clarissa Hjalmarsson, BA ${ }^{\dagger}$; Eddy Lang, MD CM; \\ Adrian Boyle, BM, MPhil, MD`; Paul Atkinson, MB, MA ${ }^{\S}$
}

\section{INTRODUCTION}

\section{Paul Atkinson (@eccucourse)}

This series of editorials will provide CFEM readers with an opportunity to hear differing perspectives on topics pertinent to the practice of emergency medicine. The debaters have been allocated opposing arguments on topics on which there is some controversy or perhaps scientific equipoise.

We continue with the topic of screening for domestic violence (DV) or intimate partner violence (IPV) in the emergency department (ED), a source of debate and discussion among health advocates, social workers, police, and clinicians. We know that patients, usually women, who have been assaulted by a partner often present to the ED for medical treatment. Is it the role of the ED staff to screen all patients, or even groups of high-risk patients, in the hope of uncovering those who have been assaulted but who do not volunteer that information? Would such an approach help these patients access appropriate support services, or might the anticipation of probing questions prevent some from seeking care in the first place? Do DV/IPV screening programs help victims escape their violent environment, or might it exacerbate the situation without the appropriate follow-up and support?

Nikita Arora and Eddy Lang from the University of Calgary propose that ED screening for DV/IPV is appropriate and can be effective, and Clarissa Hjalmarsson and Adrian Boyle from the University of Cambridge respond that while identifying patients who have experienced DV/IPV is important, routine screening is not the way to go about it.

Readers can follow the debate on Twitter and vote for either perspective, by going to @CJEMonline or searching \#CJEMdebate.

\section{\#DOMESTICVIOLENCE FOR SCREENING}

\section{Nikita Arora and Eddy Lang (@EddyLang1)}

\section{Capturing a missed opportunity-domestic violence in the $E D$}

Domestic violence refers to any physical, sexual, emotional, and financial abuse; mistreatment; or neglect of adults or children in their intimate, family, or dependent relationships. ${ }^{1}$ Statistics Canada reported that IPV alone accounted for $30 \%$ of all police-reported violent crimes in Canada in 2017, affecting 96,000 people aged 15 to 89 years, not including family violence targeting seniors and children that would further increase these numbers. ${ }^{2}$ The health care system, and the ED specifically, is a point of opportunity to screen for DV. Evidence suggests that patients who have experienced DV are more likely to seek health care services compared with non-victimized patients ${ }^{3}$ and that health care providers (HCP) are often the first contact for survivors of DV. ${ }^{4}$ Furthermore, women often identify HCPs as the professionals they

From *Department of Community Health Sciences, University of Calgary; †Clinical School, Cambridge University; $¥$ Department of Emergency Medicine, University of Calgary; §Department of Emergency Medicine, Cambridge University Hospitals Foundation Trust; and the IDepartment of Emergency Medicine, Dalhousie University, Saint John Regional Hospital.

Correspondence to: Dr. Paul Atkinson, Emergency Department, Saint John Regional Hospital, 400 University Avenue, Saint John, New Brunswick, E2L 4L4; Email: paul.atkinson@dal.ca

(C) Canadian Association of Emergency Physicians 
would trust with the disclosure of violence. ${ }^{4}$ For these reasons, universal or routine screening for DV in EDs is an important approach to identify current or former victims to offer interventions to improve health outcomes.

Domestic violence identification. As per the Cochrane review on DV screening in health care settings, ${ }^{5}$ there is moderate-certainty evidence that routine screening increases DV identification, specifically in EDs (OR 2.72 [1.03-7.19]). Similarly, observational studies confirm that the number of disclosures received by HCPs correlates with the number of women asked ${ }^{6}$ and that universal screening achieves an increase in DV identification. ${ }^{7}$ Furthermore, observational literature demonstrates that providing DV training to HCPs can increase rates of routine inquiry and disclosure rates. ${ }^{7}$ Some do argue that asking all-comers to the ED about DV would not qualify as "screening" for various reasons: 1) screening is traditionally a practice to identify the potential for a condition occurring/a condition early in its disease course, but in the case of DV, it has already occurred; and 2) DV is not a condition or disease, but a complex social circumstance. We propose that physicians move past this strict biomedical model of health and the academic exercise of determining what the definition of "screening" truly is. Instead, we encourage HCPs to routinely incorporate social determinants of health into assessments, especially for issues like DV that are highly prevalent in our patient populations, impact our patients' physical and mental health considerably, and are currently inadequately addressed by the health care system. Equipped with the important knowledge of how DV negatively impacts our patients, we need to be bold enough to change our definitions to provide better health care, instead of tailoring our health care to our definitions.

Intervention studies. Literature on DV screening in randomized controlled trials (RCTs) does not demonstrate an improvement in patient-important outcomes, an issue that is often used to oppose screening. However, it is important to note that because DV screening, in particular, is often paired with no follow-up or inadequate interventions $^{8,9}$ in the current health care environment, it is unsurprising that there will be no benefit found as a result. Unlike programs such as colorectal cancer screening, in which positive screens are automatically referred to further investigation and adequate treatment, this is often not the case for DV.
Groups like the United States Preventive Services Task Force (USPSTF) argue that studies demonstrating benefit from interventions in populations identified in health care settings indirectly support the value of screening in these settings. ${ }^{9}$ Interventions vary across studies and include cognitive-behavioural therapy, video education, cues to a patient's provider, brief counselling by family doctor, grouped DV and relapse prevention therapy, home visitation, and behavioural interventions. Beneficial outcomes include an improvement in coping skills such as self-efficacy, ${ }^{10}$ improved knowledge and use of resources, ${ }^{10}$ a reduction in mental health symptoms, ${ }^{11}$ decreased DV recurrence, ${ }^{12}$ pregnancy coercion, ${ }^{13}$ very preterm birth, and very low birth weight. ${ }^{12}$ Without identification, DV victims are far less likely to benefit from these interventions.

Screening acceptability-patients. The Cochrane review ${ }^{5}$ examines a number of RCTs that evaluated the harms of DV screening in health care settings including EDs. The majority of women in these studies agreed that "health care providers should routinely ask all women about difficulties in home life and relationships" and found no adverse effects of screening. High acceptability in patients did not vary by abuse status. These studies rebut the idea that DV may be too sensitive of an issue for the ED. Moreover, many sensitive issues are often tackled in the ED if they are the underlying cause of a patient's poor health status or presenting health complaint, such as mental health or reproductive health concerns-DV is no different. One RCT even found screening to be associated with higher patient satisfaction with care. Observational studies confirm these results, even among women who may admit feeling uncomfortable with screening. ${ }^{14}$ To optimize acceptability, however, there may be a role for future research regarding the best method for screening (e.g., in person, computer, and written). It is important to note that interventions following screening must provide resources that meet women where they are in their journey with violence, in order to minimize harm, recognizing that most women may not wish to leave their violent partners upon disclosure.

Screening acceptability-HCPs. The literature related to HCP acceptability is varied. Some studies demonstrate health care workers' support for DV screening, ${ }^{15}$ with the ED identified as an appropriate setting for screening. Other studies identify barriers that need to be addressed 
for DV screening to be effective. These barriers include a lack of privacy, time constraints, not knowing how to ask, frustration when women remain unsafe, and confidentiality concerns. ${ }^{15}$ Given that acceptability is so high in patients, it may be prudent to address provider discomfort by administering improved training regarding the management of positive screens and referral processes to community supports. Moreover, workload concerns are especially important to acknowledge in the ED; however, considering that DV-involved patients utilize health care resources, including the ED, more than average, it is reasonable to implement a system that aims to decrease this use by intervening on the underlying etiology. Such an intervention has the potential to reduce future patient morbidity and, thus, decrease ED workload over the long term by decreasing health care utilization. Further, some organizations such as the World Health Organization (WHO) recommend screening based on a list of signs/symptoms ${ }^{8}$; however, this would likely increase the workload even more, as staff would have to remember the criteria for screening, instead of just screening all-comers.

In conclusion, DV is an important health issue that the health care system is currently markedly poor at addressing. Evidence suggests that DV screening in the ED increases DV identification, and health care-based interventions in those identified can improve patientimportant outcomes. Although HCPs may feel uncomfortable with the issue, evidence suggests patient acceptability is high, and there are minimal adverse effects of screening. As such, we urge Canadian EDs to take steps to improve provider comfort with screening and knowledge of community supports, in order to ultimately implement effective universal screening, and thereby display the courage to prioritize a difficult, but critical, determinant of women's and family health in Canada.

\section{\#DOMESTICVIOLENCE AGAINST SCREENING}

\section{Clarissa Hjalmarsson (@clarryhj) and Adrian Boyle (@DrAdrianBoyle)}

\section{"It is important not to do the wrong thing for the right reason"}

Domestic or intimate partner violence (IPV) is a public health and human rights issue that affects around one in four women and one in five men during their lifetimes. Despite this prevalence, it frequently passes undetected. Domestic violence is associated with multiple poor outcomes such as physical injury, chronic physical, complaints, unwanted pregnancy or poor pregnancy outcomes, substance misuse, and death. ${ }^{17}$ For a multitude of reasons, victims typically only disclose IPV after prolonged periods of abuse, and clinicians frequently fail to ask. Individuals who disclose abuse can be offered effective interventions such as advocacy. Clinicians have a professional and ethical legal responsibility to facilitate disclosure. ${ }^{18}$

Emergency physicians are frequently well placed to identify victims, and screening for domestic abuse in the ED appears to provide an intuitive answer to helping victims of IPV. However, routine enquiry for IPV is not screening and may, in fact, be unacceptable and ineffective and potentially cause harm to vulnerable individuals.

Routine enquiry for domestic abuse is not screening. In medicine, screening is a process used to identify the possible presence of a detrimental condition in (asymptomatic) individuals. Wilson's widely recognized criteria for screening emphasize that a test should be "easy to perform and interpret, acceptable, accurate, reliable, sensitive, and specific." ${ }^{19}$ For example, asymptomatic women in the United Kingdom aged 50-70, who are statistically at higher risk of breast cancer, are offered a mammogram every three years. The benefits and harms are explained prior to screening; any resulting scans are objectively assessed; and patients with positive screening results are offered further tests and evidence-based medical interventions. ${ }^{20}$ While these standardized procedures may be appropriate for conditions such as breast cancer or hypertension, "screening" for IPV is a different matter. IPV is not a disease with well-defined and predictable outcomes, but rather a complex and highly variable social phenomenon that may comprise a single incident or pattern of behaviour. Routine enquiry for IPV is not, therefore, a "screening" program ${ }^{17}$ but a series of potentially highly delicate questions with unpredictable outcomes.

Screening is unacceptable. Research into the acceptability of routine enquiry has suggested that up to $57 \%$ of women find it unacceptable, but historic studies suggest that two-thirds of doctors $(67 \%)$ and almost one-half of nurses (47\%) are not in favour. ${ }^{21,22,23}$ More importantly, screening for abuse was found to be significantly less acceptable to women who have suffered IPV in the last year, compared with women who 
have not suffered from IPV or, interestingly, who were lifetime sufferers. ${ }^{24}$ These findings echo similar work conducted by a phone interview by Sachs et al., who demonstrated that women who suffer from abuse are less likely to support screening. ${ }^{25}$ A process of routine enquiry may, therefore, be least acceptable to those it is intended to help most.

The ED presents a number of barriers to disclosure, not least the substantial differential in power/authority between clinicians and patients; one study found that higher rates of IPV disclosure are achieved if women use self-administered methods, rather than face to face. ${ }^{26}$ Mandating a standardized process of screening for all patients may exacerbate existing barriers to inquiry and disclosure, such as a lack of time, resources, and clinician (in)consistency.

Screening is ineffective. The evidence that screening improves outcomes for IPV is generally of poor quality, and recent systematic reviews of existing research into IPV remain equivocal on the question of screening. ${ }^{16}$ As Wathen and MacMillan demonstrate, there is currently no comparative data evaluating the effectiveness of screening for which the endpoint is improved outcomes for victims of IPV. ${ }^{27}$ Much of the research into ED screening focuses on an increase in referral to agencies, which is a poor measure of effectiveness ${ }^{17}$; current guidance recommends that the success of interventions should be quantified using the incidence of abuse. ${ }^{28}$ A systematic review by Nelson et al. goes further, proposing outcome measures such as improved quality of life, self-esteem, social support, and productivity among those who report IPV. ${ }^{18}$ Currently, the imposition of a screening program does not appear to be supported by evidence of effectiveness.

In addition, many EDs are poor environments for disclosing IPV. A frequent lack of privacy and the perception by patients that staff is too busy to talk ${ }^{16}$ make emergency care poorly suited to mandatory questioning on a potentially sensitive matter. Only $19 \%$ of female victims of domestic abuse approach a doctor for help, of whom $65 \%$ presented to their general practitioner (GP) rather than the ED. ${ }^{17}$ More appropriate opportunities for screening for IPV, such as obstetrics and gynecology clinics, antenatal care, and psychiatry, are already utilized and recommended by advisory bodies. ${ }^{17}$ In the ED, the desire to cast a wide net with regard to domestic violence may come at the cost of focusing time and resources on identifying those in need.
Screening could be harmful. Furthermore, screening might be harmful to the identification and treatment of IPV. Mandating that time-pressed clinicians ask a routine screening question for IPV as part of a checklist of assessments, such as drug allergies, frailty, and housing arrangements, may encourage enquiry in a way that inhibits disclosure. For an issue with the emotional and social complexities of domestic violence, asking about abuse badly or with complacency may do more harm than good and increase feelings of isolation.

There are also significant potential harms caused by screening, which are repeatedly understudied or omitted from evaluations. A BMA Board of Science report raised concerns about the multitude of risks facing vulnerable individuals who do decide to disclose IPV, including reprisal violence, psychological distress, and family disruption, particularly in the longer term. ${ }^{17}$ Nelson also highlighted the risks to victims of a loss of personal residence or financial resources, a loss of autonomy or employment, being targeted for retaliation, and a loss of control. ${ }^{29}$ While the intentions behind screening for domestic violence are admirable, blindly applying a screening program to all women in the ED rather than targeting the enquiry to individuals presenting with indicators may do more harm than good.

Alternatives to screening. There are clear arguments against implementing screening for IPV in EDs, and this decision is supported by a number of expert guideline bodies, including NICE, ${ }^{28}$ the National Screening Council, and the Royal College of Emergency Medicine, suggesting that "clinicians should be prepared to ask simple direct questions if there is any clinical suspicion." ${ }^{30}$ Nevertheless, we accept that emergency physicians should ask about IPV more often than we currently do.

We suggest an alternative, three-pronged approach for identifying IPV. First, clinicians should receive regular, high-quality training to ensure that they feel comfortable asking targeted questions about IPV. Second, EDs should offer an environment that facilitates and encourages disclosure, rather than the symbolic privacy afforded by a curtain, in which staff who suspect abuse can inquire about IPV in private. Finally, service providers should ensure that the referral pathways for victims of IPV are transparent and well-understood by clinicians and patients.

It is our duty as emergency physicians to provide the safest and most effective means possible of identifying and intervening in cases of IPV. It is important not to do the wrong thing for the right reasons. 
Keywords: Domestic violence, emergency department, screening, advocacy

Competing interests: None declared.

\section{REFERENCES}

1. Department of Justice Canada. Family Violence: Department of Fustice Canada Overview Paper. Ottawa Department of Justice Canada; 2009.

2. Burczycka M. Section 2: Police-reported intimate partner violence in Canada, 2017. Available at: https://www150. statcan.gc.ca/n1/pub/85-002-x/2018001/article/54978/02eng.htm. (accessed February 1, 2019).

3. Bonomi AE, Anderson ML, Rivara FP, Thompson RS. Health care utilization and costs associated with physical and nonphysical-only intimate partner violence. Health Serv Res 2009;44(3):1052-67.

4. Feder GS, Hutson M, Ramsay J, Taket AR. Women exposed to intimate partner violence: expectations and experiences when they encounter health care professionals: a meta-analysis of qualitative studies. Arch Intern Med 2006;166(1):22-37.

5. O'Doherty L, Hegarty K, Ramsay J, et al. Screening women for intimate partner violence in healthcare settings. Cochrane database of systematic reviews. 2015;7:CD0070007. https://doi. org/10.1002/14651858.CD007007.pub3.

6. Price S, Baird K, Salmon D. Does routine antenatal enquiry lead to an increased rate of disclosure of domestic abuse? Findings from the Bristol Pregnancy and Domestic Violence Programme. Evid Based Midwifery 2007;5(3):100-7.

7. Ramsden C, Bonner M. An early identification and intervention model for domestic violence. Australas Emerg Nurs 7 2002;5(1):15-20.

8. World Health Organization. Responding to intimate partner violence and sexual violence against women: WHO clinical and policy guidelines. World Health Organization; 2013.

9. Feltner C, Wallace I, Berkman N, et al. Screening for intimate partner violence, elder abuse, and abuse of vulnerable adults: An evidence review for the U.S. Preventive Services Task Force. Rockville (MD): Agency for Healthcare Research and Quality; 2018, report number 18-05240-EF-1.

10. Miller E, Tancredi DJ, Decker MR, et al. A family planning clinic-based intervention to address reproductive coercion: a cluster randomized controlled trial. Contraception 2016;94 (1):58-67.

11. Hegarty K, O'Doherty L, Taft A, et al. Screening and counselling in the primary care setting for women who have experienced intimate partner violence (WEAVE): a cluster randomised controlled trial. Lancet 2013;382(9888):249-58.

12. Kiely M, El-Mohandes AA, El-Khorazaty MN, Blake SM, Gantz MG. An integrated intervention to reduce intimate partner violence in pregnancy: a randomized controlled trial. Obstet Gynecol 2010;115(2 Pt 1):273-83.

13. Miller E, Decker MR, McCauley HL, et al. A family planning clinic partner violence intervention to reduce risk associated with reproductive coercion. Contraception 2011;83(3):274-80.
14. Webster J, Stratigos SM, Grimes KM. Women's responses to screening for domestic violence in a health-care setting. Midwifery 2001;17(4):289-94.

15. Power C, Bahnisch L, McCarthy D. Social work in the emergency department-implementation of a domestic and family violence screening program. Aust Soc Work 2011;64(4):537-54.

16. Department of Health. Responding to domestic abuse: A resource for health professionals. UK: Department of Health; 2017; 8,33.

17. BMA Board of Science. Domestic Abuse. June 2007, updated September 2014,.1-2;40-4.

18. Nelson HD, Nygren P, McInerney Y, Klein J US Preventive Services Task Force. Screening women and elderly adults for family and intimate partner violence: a review of the evidence for the US Preventive Services Task Force. Ann Intern Med 2004;140(5):387-96.

19. Wilson JM, Jungner G. Principles and Practice of Screening for Disease, WHO Public Health Paper 34. Geneva: WHO; 1968.

20. Marmot MG, Altman DG, Cameron DA, et al. The benefits and harms of breast cancer screening: an independent review. Br 7 Cancer 2013;108(11):2205-40.

21. Ramsay J, Richardson J, Carter YH, Davidson LL, Feder G. Should health professionals screen women for domestic violence? Systematic review. BM7 2002;325(7359):314-37.

22. Ellis JM. Barriers to effective screening for domestic violence by registered nurses in the emergency department. Crit Care Nurs Q 1999;22(1):27-41.

23. Friedman LS, Samet JH, Roberts MS, Hudlin M, Hans P. Inquiry about victimization experiences. A survey of patient preferences and physician practices. Arch Intern Med 1992;152(6):1186-90.

24. Boyle A, Jones PB. The acceptability of routine inquiry about domestic violence towards women: a survey in three healthcare settings. Br 7 Gen Pract 2006;56(525):258-61.

25. Sachs CJ, Koziol-McLain J, Glass N, Webster D, Campbell J. A population-based survey assessing support for mandatory domestic violence reporting by health care personnel. Women Health 2002;35(2-3):121-33.

26. Nelson HD, Bougatsos C, Blazina I. Screening women for intimate partner violence: a systematic review to update the US Preventive Services Task Force recommendation. Ann Intern Med 2012;156(11):796-808.

27. Wathen CN, MacMillan HL. Interventions for violence against women: scientific review. $\mathcal{F} A M A$ 2003;289(5):589600.

28. NICE Guidelines: Domestic Violence and Abuse. London: National Institute for Health and Care Excellence; 2016, 11-12.

29. Nelson HD, Bougatsos C, Blazina I. Screening women for intimate partner violence: a systematic review to update the US Preventive Services Task Force recommendation. Ann Intern Med 2012;156(11):796-808.

30. College of Emergency Medicine, Clinical Effectiveness Committee. Guideline for the recognition and management of domestic violence in emergency departments. UK: College of Emergency Medicine, Clinical Effectiveness Committee; 2010. 